\title{
BMJ Open Identification of factors associated with high-cost use of inpatient care in chronic kidney disease: a registry study
}

\author{
P. Marcin Sowa (1) , ,2 Sree K. Venuthurupalli, ${ }^{1,3}$ Wendy E. Hoy (1) ,1,3 \\ Jianzhen Zhang, ${ }^{1,3}$ Anne Cameron, ${ }^{1,3}$ Helen G. Healy, ${ }^{1,3}$ Luke B. Connelly ${ }^{1,2,4}$
}

To cite: Sowa PM, Venuthurupalli SK, Hoy WE, et al. Identification of factors associated with high-cost use of inpatient care in chronic kidney disease: a registry study. BMJ Open 2021;11:e049755. doi:10.1136/ bmjopen-2021-049755

- Prepublication history and additional supplemental materia for this paper are available online. To view these files, please visit the journal online. (http://dx.doi.org/10.1136/ bmjopen-2021-049755)

Received 01 February 2021 Accepted 27 July 2021

Check for updates

(c) Author(s) (or their employer(s)) 2021. Re-use permitted under CC BY-NC. No commercial re-use. See rights and permissions. Published by BMJ.

${ }^{1}$ NHMRC Chronic Kidney Disease Centre of Research Excellence, The University of Queensland, Herston, Queensland, Australia ${ }^{2}$ Centre for the Business and Economics of Health, The University of Queensland, St Lucia, Queensland, Australia ${ }^{3}$ Faculty of Medicine, The University of Queensland, Herston, Queensland, Australia ${ }^{4}$ Department of Sociology and Business Law, The University of Bologna, Bologna, Italy

Correspondence to

Dr P. Marcin Sowa;

m.sowa@uq.edu.au

\section{ABSTRACT}

Objective To explore factors behind inpatient admissions by high-cost users (HCUs) in pre-end-stage chronic kidney disease (CKD).

Design Retrospective analysis of CKD.QLD Registry and hospital admissions of the Queensland Government Department of Health recorded between 1 July 2011 and 30 June 2016.

Setting Queensland public and private hospitals. Participants 5096 individuals with CKD who consented to the CKD.QLD Registry via 1 of 11 participating sites. Main outcomes Associations of HCU status with patient characteristics, pathways and diagnoses behind hospital admissions at 12 months.

Results Age, advanced CKD, primary renal diagnosis, cardiovascular disease and hypertension were predictors of the high-cost outcome. HCUs were more likely than non-HCUs to be admitted by means of episode change (relative risk: $5.21 ; 95 \% \mathrm{Cl} 5.02$ to 5.39), 30-day readmission $(2.19 ; 2.13$ to 2.25$)$, scheduled readmission $(1.29 ; 1.11$ to 1.46$)$ and emergency $(1.07 ; 1.02$ to $1.13)$, for diagnoses of the nervous $(1.94 ; 1.74$ to 2.15$)$, circulatory $(1.24 ; 1.14$ to 1.34$)$ and respiratory $(1.2 ; 1.03$ to 1.37$)$ systems and other factors influencing health status $(1.92 ; 1.74$ to 2.09$)$.

Conclusions The high relevance of episode change and other factors influencing health status revealed that a substantial part of excess demand for inpatient care was associated with discordant conditions often linked to frailty, decline in psychological health and social vulnerability. This suggests that multidisciplinary models of care that aim to manage discordant comorbidities and address psychosocial determinants of health, such as renal supportive care, may play an important role in reducing inpatient admissions in this population.

\section{INTRODUCTION}

Kidney failure is associated with considerably increased need for healthcare services compared with the general population. ${ }^{1-4}$ Mulitmorbidity, defined as the presence of two or more associated chronic health conditions, is an important driver enhancing the complex needs of patients with chronic kidney disease (CKD). ${ }^{5}$ Concordant conditions, that is comorbidities that are either causes (diabetes, hypertension) or consequences (cardiac

\section{Strengths and limitations of this study}

The large, pre-dialysis patient registry, linked with a comprehensive hospitalisations data set, allows a wide-ranging exploration of factors associated with patients with chronic kidney disease (CKD) who are admitted to hospitals.

- The study focuses on the first year of patients coming into specialist care, which is a less explored area compared with the end-stage or near-end-stage kidney disease.

- The nature of the study is exploratory and hypothesis-generating.

- Undiagnosed cases and patients not presenting to Queensland public renal practice clinics are not included in this study.

- Study enrolment emphasises relatively advanced CKD that warrants specialist care, which limits its generalisability to a full spectrum of CKD.

disease, anaemia) of CKD, are well understood with both preventive and management strategies in place. Less studied is the impact and interrelation of discordant conditions such as cancer, dementia, arthritis and frailty, which also significantly contribute toward poor outcomes in CKD population, especially in hospitalised patients. ${ }^{67}$ These comorbidities lead to excessive hospital admissions and readmissions, prolonged hospital stay, rehabilitation, displacement from home and higher healthcare expenditure. Improving our understanding of the distinguishing characteristics of those patients with CKD who are high-cost users (HCUs), ways through which they enter the hospital system, and diagnoses leading to admissions that differentiate them from other users, is an essential next step in reducing the health system burden of kidney disease. ${ }^{8}$ Pathways to admissions, emergency and readmission, in particular, offer useful analytical angles that allow identification and prevention of avoidable hospitalisations at the point of entry. ${ }^{9-11}$ 
Our aim was to identify and describe HCUs in an attempt to better characterise the demographic profiles, health needs and pathways to inpatient care, associated with the high-cost outcome. With the broader objective of informing the development and targeting of models of care that seek to address the specific risk factors and needs, and incorporate strategies to prevent complications in CKD care pathways. Recent years have seen the emergence of new models of care designed to benefit patients with kidney disease at pre-dialysis stages. ${ }^{12}$ Robust programmes such as Kidney Supportive Care ${ }^{13}$ have been demonstrated to offer improved outcomes and quality of care to patients as well as economic benefits of lowering long-term pressures on the healthcare system due to reduced need for costly downstream healthcare services. ${ }^{14}$ Insights from our study will guide proactive implementation of these models of care, in patient groups identified as likely to become high users of hospital care in a short to mid-term horizon.

\section{METHODS \\ Data}

Data on patient demographics (age, sex, indigenous status) and health status (estimated glomerular filtration rate (eGFR), renal diagnosis, comorbidities, body mass index) were extracted from the CKD. QLD Registry, a statewide surveillance programme enrolling patients diagnosed with CKD. ${ }^{15}$ The medical records for these patients were retrieved from the electronic systems of Queensland Government Department of Health, capturing episodes, pathways, diagnoses, procedures, costs and deaths during hospital admissions within the state between 1 July 2011 and 30 June $2016 .{ }^{16}$ The costs were approximated relative to resource intensity of care using Queensland Health activity-based funding guidelines. ${ }^{17}$ The combined data set was inspected for missing variables and conflicting information between the two sources. Reaching the point of kidney failure was interpreted as study exit. The determination of the study sample is presented in figure 1 .

\section{Analyses}

Following a convention accepted in the health services research literature, ${ }^{18}$ we defined and retrospectively identified HCUs as the top $10 \%$ of the study sample with respect to inpatient costs incurred during the 12-month period. We compared the HCUs against those who did not classify as HCU (henceforth referred to as the non-HCUs). To allow further insights into the sample, we presented the non-HCUs as two subgroups: those who were never admitted and those admitted at least once. The 1-year horizon served to maintain the accuracy of variables representing time-variant characteristics, such as CKD stage and comorbidities, recorded at the time of enrolment. We summarised patient characteristics (gender, indigenous status, age, the BMI, kidney function, primary renal diagnosis and comorbidities), selected outcomes at 12 months (frequent admission status, dialysis status,

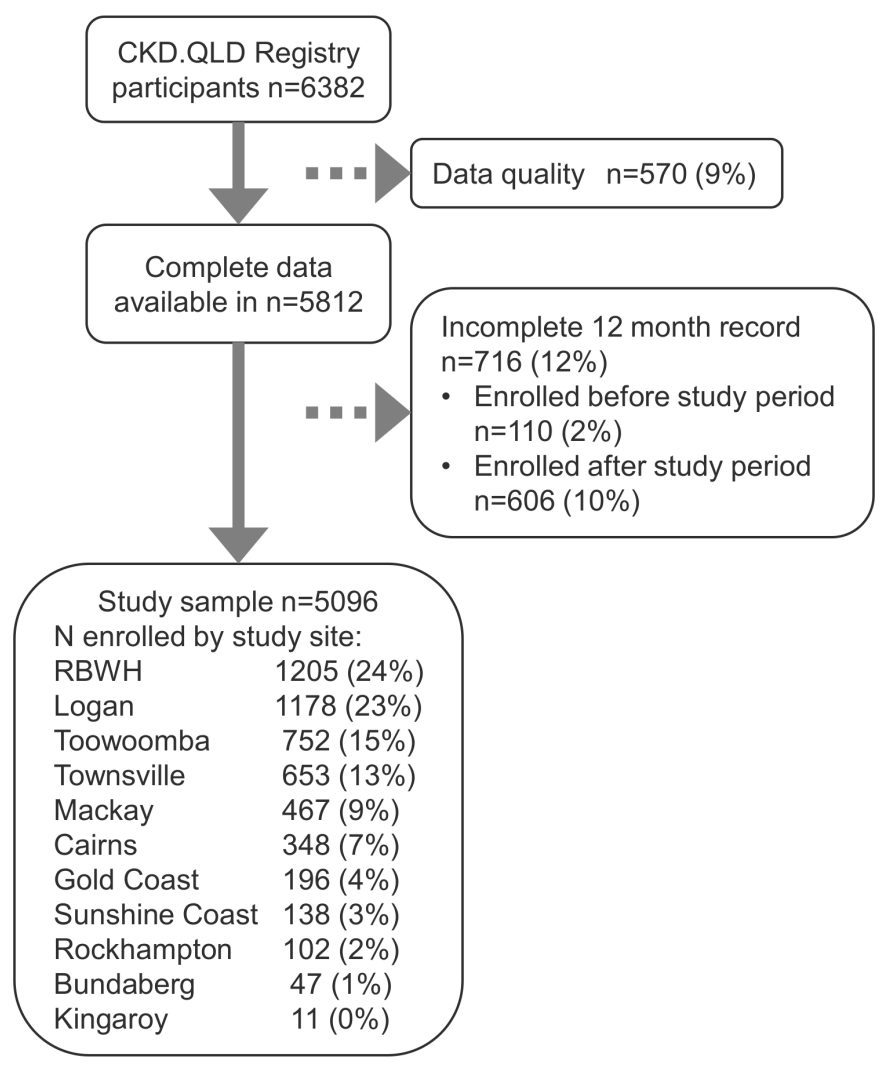

Figure 1 Selection of the study sample.

renal replacement therapy, death, and the cost outcome) using descriptive statistics, and used t-tests and Pearson's $\chi^{2}$ to compare HCUs and non-HCUs. We used logistic regression to determine which patient characteristics known at the time of study enrolment predicted the HCU status at 12 months, expressed as odds ratios (ORs). We calculated relative risks (RRs) of hospitalisation pathways (elective, day-hospital, readmission and by source of referral) to find systematic differences in admission patterns between the groups. Finally, we computed RRs for major diagnostic categories (MDCs), which group International Classification of Disease (ICD) codes into 22 mutually exclusive diagnosis areas, to identify diagnoses that were relatively more common in the HCUs, and reported the major MDCs that explained at least $75 \%$ of the cost on record. Supplementary material presents means, SD and frequencies of costs by hospitalisation pathway (online supplemental table S1) and by MDC (online supplemental table S2). Expenditures occurring in different years were made comparable by adjusting for the inflation rate ${ }^{19}$ and reported as 2015-2016 Australian dollars.

\section{RESULTS}

The 90th percentile of the distribution of individual hospital expenditures in the final study sample $(n=5096)$ was A $\$ 28,191 ; 509$ (10\%) individuals above this threshold qualified as HCUs. Those who were admitted at least once but did not qualify as HCUs ( $\mathrm{n}=2139)$ accounted for $42 \%$ 
of the sample. The remaining study participants $(n=2448$; $48 \%$ ) did not have a record of admission (table 1 ).

\section{Characteristics and selected outcomes}

Comparing HCUs versus non-HCUs (table 1), we found no statistically significant differences in the proportions of men (55.4\% vs $53.7 \%$; $=0.475)$ and indigenous Australians $(8.5 \%$ vs $7.3 \%$; $=0.384)$. The HCU group had greater proportions of patients in age bands above 60 years, which was reflected in the statistically significant difference in mean age (69.1 vs 64.5 years; $\mathrm{p}=0.000$ ). The body mass index was not statistically associated with hospital utilisation. HCUs had more advanced CKD, with $48.1 \%$ enrolling at stages 4 and 5, compared with $32.8 \%$ in non-HCUs $(\mathrm{p}=0.000)$. Differences in eGFR means reflected this pattern, with the mean eGFR of HCUs 8.2 $\mathrm{mL} / \mathrm{min} / 1.73 \mathrm{~m}^{2}$ points lower than in the other group $(\mathrm{p}=0.000)$. A comparison of primary renal diagnosis categories revealed that diabetic nephropathy was more common in the HCU group $(33.2 \%$ vs $23.2 \%$ in non$\mathrm{HCU}$ ), while glomerulonephritis and renovascular disease were less common $(p=0.000)$. There was a clear pattern of greater prevalence and higher number of comorbidities among the HCUs compared with non-HCUs. The rates were $63.6 \%$ versus $50.2 \%$ for diabetes $(\mathrm{p}=0.000), 71.3 \%$ versus $46.5 \%$ for cardiovascular disease $(\mathrm{p}=0.000)$ and $86.3 \%$ versus $79.8 \%$ for hypertension $(\mathrm{p}=0.003)$. Multiple comorbidity status, defined as combinations of the above conditions, followed a similar pattern $(\mathrm{p}=0.000)$.

HCU status was associated with high chances of being frequently admitted, with $51.1 \%$ qualifying as top $10 \%$ frequent users ( $\geq 5$ inpatient episodes in 12 months; $\mathrm{p}=0.000$ ). HCUs had significantly higher admission rates for the purpose of dialysis $(\mathrm{p}=0.000)$, but the rates were very low overall, $1.4 \%$ in HCUs and $0.3 \%$ in others. Renal replacement therapy was reached by $7.3 \%$ of HCUs, compared with $2.9 \%$ in non-HCU $(\mathrm{p}=0.000)$. The proportion of patients who died within the first year of the study was considerably higher in the HCU group $(19.5 \%$ vs $2.7 \%$ in non-HCU; $\mathrm{p}=0.000)$.

Factors known at the time of enrolment that predicted the HCU status at 12 months were advanced CKD (stage 4 OR 1.84; $\mathrm{p}=0.008$ and stage 5, OR 1.82; $\mathrm{p}=0.036$ ), diabetic nephropathy $(1.61 ; \mathrm{p}=0.009)$ and selected other renal diagnoses $(1.58 ; \mathrm{p}=0.006)$, cardiovascular conditions $(2.48 ; \mathrm{p}=0.000)$ and hypertension $(1.53 ; \mathrm{p}=0.028)$ (table 2). Individuals with primary renal diagnosis of glomerulonephritis or renovascular were less likely to be HCUs, compared with diabetic nephropathy and other primary renal diagnoses. Participants age 40 years or less were less likely to reach the HCU status $(0.45 ; \mathrm{p}=0.044)$, but within those 40 years old or above age was not a statistically significantly predictor of the outcome.

\section{Pathways}

Hospitalisation pathways were analysed across 7315 admissions, of which $2836(38.8 \%)$ were in the top $10 \%$ costliest users. HCUs systematically differed from non-HCUs across all analysed pathways to admission (figure 2). Episode change, either within the admitting institution $(48.2 \%)$ or in transfer from another hospital (51.8\%), stood out as an admission pathway. The RR of this event was 5.21 (95\% CI 5.02 to 5.39) in the HCU group relative to non-HCU. Episode change accounted for $17 \%$ of all admissions in the HCU group, compared with $3 \%$ in the non-HCU. HCUs were also more likely to be readmitted within 30 days of a previous admission (2.19; 2.13 to 2.25$)$, as a scheduled readmission $(1.29 ; 1.11$ to 1.46$)$ or as a result of an emergency presentation $(1.07 ; 1.02$ to 1.13). HCUs' admissions were less likely to be for elective procedures $(0.58 ; 0.52$ to 0.65$)$, through the outpatient department $(0.66 ; 0.58$ to 0.74$)$, by general practitioner (GP) or specialist referral $(0.65 ; 0.52$ to 0.78$)$ or to take the form of day-hospital episodes $(0.66 ; 0.58$ to 0.73$)$.

\section{Diagnoses}

The number of admissions per person in the HCU group was 2.66 times that of admitted non-HCU (table 3). The top seven MDCs accounted for $77.1 \%$ of inpatient expenditures. Relative to others, HCUs were at an increased risk of admissions due to issues of the nervous system (RR: 1.94; $95 \%$ CI 1.74 to 2.15 ), factors influencing health status (FIHS) (1.92; 1.74 to 2.09), circulatory (1.24; 1.14 to 1.34 ) and respiratory system (1.2; 1.03 to 1.37$)$. HCUs were at a lower risk of admissions caused by digestive system issues $(0.71 ; 0.56$ to 0.87$)$ or other MDCs $(0.73$; 0.66 to 0.81 ). Key FIHS that distinguished HCU from non-HCU involved the use of rehabilitation procedures (Z50, $47.4 \%$ vs $20.8 \%$, respectively) and problems related to medical facilities and other healthcare (Z75, $13.5 \%$ vs $1.5 \%$, respectively).

\section{DISCUSSION}

The expenditures in our sample showed a considerable degree of concentration, with $10 \%$ high-end users responsible for $65.2 \%$ of recorded expenditures. While high cost and admission frequency are correlated, only half of the HCUs qualified as frequent users, which illustrates a complication in the relationship between these two criteria. Frequency of contact with healthcare explains some, but not all, of the resulting costs. It follows that there are other important factors, such as types and severity of conditions, and perhaps differences in chronic disease management, that account for the remaining part of the expenditure variation.

We found good agreement between descriptive statistics summarising our sample and the HCU prediction model. CKD stage and key comorbidities were confirmed as predictors of health and utilisation outcomes previously documented in studies from the UK, ${ }^{1}$ Australia, ${ }^{2}$ Germany, ${ }^{3}$ the USA, ${ }^{4}$ Italy, ${ }^{20}$ Sweden $^{21}$ and Canada. ${ }^{22}$ We found age to be a more nuanced factor, showing the statistical association of a lower probability of HCU outcome in younger patients, but failing to explain cost variation for those over 40 . Neither descriptive nor 
Table 1 Patient characteristics at the time of study consent and selected 12-month outcomes

\begin{tabular}{|c|c|c|c|c|}
\hline & \multicolumn{2}{|l|}{ Non-HCU } & \multirow[b]{2}{*}{$\mathrm{HCU}$} & \multirow[b]{2}{*}{ P value* } \\
\hline & Not admitted & Admitted & & \\
\hline $\mathrm{n}$ & 2448 & 2139 & 509 & \\
\hline Male, n (\%) & 1319 (53.9) & 1146 (53.6) & $282(55.4)$ & 0.475 \\
\hline Indigenous, n (\%) & $125(6.5)$ & $133(8.1)$ & $32(8.5)$ & 0.384 \\
\hline Age, mean years (SD) & 63 (15.9) & $66.1(15)$ & $69.1(13.1)$ & 0.000 \\
\hline Age band, n (\%) & & & & 0.000 \\
\hline$<40$ & $260(10.6)$ & $154(7.2)$ & $15(3)$ & \\
\hline $40-49$ & 235 (9.6) & $163(7.6)$ & $32(6.3)$ & \\
\hline $50-59$ & 381 (15.6) & 295 (13.8) & 62 (12.2) & \\
\hline $60-69$ & $605(24.7)$ & $519(24.3)$ & $130(25.5)$ & \\
\hline $70-79$ & $671(27.4)$ & $659(30.8)$ & $167(32.8)$ & \\
\hline$\geq 80$ & $296(12.1)$ & 349 (16.3) & $103(20.2)$ & \\
\hline BMI, mean (SD) & $31.3(7.8)$ & $31.1(7.7)$ & $31.4(8.8)$ & 0.788 \\
\hline BMI group & & & & 0.425 \\
\hline$\leq 18.5$ & $16(1)$ & $19(1.3)$ & $5(1.5)$ & \\
\hline $18.5-25$ & 258 (15.7) & 231 (16.3) & 63 (19.2) & \\
\hline $25-30$ & $484(29.5)$ & 415 (29.3) & $90(27.4)$ & \\
\hline$>30$ & 885 (53.9) & $752(53.1)$ & $170(51.8)$ & \\
\hline eGFR, mean (SD) & $44.8(21.9)$ & $37.3(20.5)$ & $33.2(17.8)$ & 0.000 \\
\hline CKD stage, n (\%) & & & & 0.000 \\
\hline $1-2$ & $509(21.6)$ & 261 (12.8) & $40(8.3)$ & \\
\hline 3 & $1224(52)$ & $963(47.1)$ & $210(43.6)$ & \\
\hline 4 & $521(22.1)$ & 585 (28.6) & $178(36.9)$ & \\
\hline 5 & $101(4.3)$ & 236 (11.5) & 54 (11.2) & \\
\hline Primary renal diagnosis, n (\%) & & & & 0.000 \\
\hline Diabetic nephropathy & 472 (20.9) & $503(26.1)$ & $157(33.2)$ & 0.000 \\
\hline Glomerulonephritis & $338(14.9)$ & $227(11.8)$ & $40(8.5)$ & 0.002 \\
\hline Renovascular & 667 (29.5) & $634(32.8)$ & $134(28.3)$ & 0.231 \\
\hline Other & $787(34.8)$ & $567(29.4)$ & $142(30)$ & 0.319 \\
\hline \multicolumn{5}{|l|}{ Comorbidities, n (\%) } \\
\hline Diabetes & 952 (46.5) & $974(54.5)$ & $262(63.6)$ & 0.000 \\
\hline CVD & $599(38)$ & $761(56.5)$ & $229(71.3)$ & 0.000 \\
\hline Hypertension & $1376(78.7)$ & $1208(81)$ & $309(86.3)$ & 0.003 \\
\hline \multicolumn{5}{|l|}{ Multi-comorbidity status, $\mathrm{n}(\%)$} \\
\hline Diabetes and CVD & $327(13.4)$ & $444(20.8)$ & $147(28.9)$ & 0.000 \\
\hline Diabetes and hypertension & $598(24.4)$ & $633(29.6)$ & $178(35)$ & 0.000 \\
\hline CVD and hypertension & $502(20.5)$ & $650(30.4)$ & $209(41.1)$ & 0.000 \\
\hline Diabetes, CVD and hypertension & $279(11.4)$ & $393(18.4)$ & $135(26.5)$ & 0.000 \\
\hline \multicolumn{5}{|c|}{ Selected outcomes at 12 months, $\mathrm{n}(\%)$} \\
\hline$\geq 5$ inpatient admissions & $0(0)$ & $138(6.5)$ & $260(51.1)$ & 0.000 \\
\hline Admitted for dialysis $†$ & $0(0)$ & $13(0.6)$ & $7(1.4)$ & 0.000 \\
\hline Renal replacement therapy & $0(0)$ & $135(6.3)$ & $37(7.3)$ & 0.000 \\
\hline Death & $27(1.1)$ & $95(4.4)$ & 99 (19.5) & 0.000 \\
\hline \multicolumn{5}{|l|}{ Selected cost outcome statistics } \\
\hline $\mathrm{A} \$$, mean $(\mathrm{SD})$ & $0(0)$ & 8091 (7155) & 63828 (42 200) & 0.000 \\
\hline
\end{tabular}




\begin{tabular}{lcccc} 
& Non-HCU & & \\
\cline { 2 - 3 } & Not admitted & Admitted & HCU & P value* \\
\hline A \$, median (IQR) & $0(0)$ & $5734(9656)$ & $588(34068)$ & 0.000 \\
Skewness & 0 & 1.0024 & 2.761 & \\
\hline
\end{tabular}

The non-HCU group comprises of those never admitted, and those admitted at least once. The three groups reported in the table are mutually exclusive.

Statistical significance denoted in bold for $p$-values $<0.05$.

*T-test (continuous), Pearson's $\chi^{2}$ (categorical) or Wilcoxon rank-sum test (medians) comparing HCU versus non-HCU. †Admissions with DRG code L61Z or L68Z.

BMI, body mass index; CVD, cardiovascular disease; DRG, diagnosis-related group; eGFR, estimated glomerular filtration rate; HCU, highcost user.

analytical approaches confirmed the indigenous status as a predictor of the HCU outcome in our sample. This was contrary to our expectations based on the known underlying gaps in health and healthcare between indigenous and non-indigenous Australians. ${ }^{23}$

Of the kidney disease characteristics, we found statistical evidence linking primary renal diagnosis to inpatient

Table 2 Logistic regression predicting HCU status using information available at the time of study enrolment

\begin{tabular}{|c|c|c|c|}
\hline & OR & $95 \% \mathbf{C l}$ & $P$ value \\
\hline Male & 0.86 & 0.68 to 1.1 & 0.231 \\
\hline Indigenous & 1.25 & 0.7 to 2.23 & 0.461 \\
\hline \multicolumn{4}{|l|}{ Age band } \\
\hline$<40$ & 0.45 & 0.21 to 0.98 & 0.044 \\
\hline $40-49$ & 1.10 & 0.65 to 1.86 & 0.722 \\
\hline $50-59$ & 1.17 & 0.79 to 1.75 & 0.431 \\
\hline $60-69$ & Ref. & & \\
\hline $70-79$ & 0.97 & 0.7 to 1.35 & 0.870 \\
\hline$\geq 80$ & 1.27 & 0.88 to 1.86 & 0.205 \\
\hline \multicolumn{4}{|l|}{ CKD stage } \\
\hline $1-2$ & Ref. & & \\
\hline 3 & 1.20 & 0.78 to 1.84 & 0.401 \\
\hline 4 & 1.84 & 1.18 to 2.88 & 0.008 \\
\hline 5 & 1.82 & 1.04 to 3.19 & 0.036 \\
\hline \multicolumn{4}{|l|}{ Primary renal diagnosis } \\
\hline Renovascular & Ref. & & \\
\hline Diabetic nephropathy & 1.61 & 1.13 to 2.3 & 0.009 \\
\hline Glomerulonephritis & 1.20 & 0.71 to 2.03 & 0.501 \\
\hline Other & 1.58 & 1.14 to 2.19 & 0.006 \\
\hline \multicolumn{4}{|l|}{ Comorbidities } \\
\hline Diabetes & 1.11 & 0.81 to 1.52 & 0.509 \\
\hline CVD & 2.48 & 1.87 to 3.28 & 0.000 \\
\hline Hypertension & 1.53 & 1.05 to 2.25 & 0.028 \\
\hline Constant & 0.02 & 0.01 to 0.05 & 0.000 \\
\hline
\end{tabular}

Statistical significance denoted in bold for $p$-values $<0.05$.

CKD, chronic kidney disease; CVD, cardiovascular disease. care utilisation. Specifically, patients whose renal disease was classified as renovascular or glomerulonephritis had lower odds of being HCUs compared with patients diagnosed with diabetic nephropathy or other types of disease. Prior evidence suggests a role for renal disease type in explaining variation in health outcomes, ${ }^{24}$ but to our knowledge, this is the first time it has been linked to utilisation outcomes. This finding should invite further explorations of healthcare utilisation with respect to subtypes of renal disease, including those of genetic origin. ${ }^{25}$

HCUs had higher rates of admissions due to issues concerning the circulatory, nervous and respiratory systems and other FIHS, but the RR of an episode directly related to kidney disease was not statistically different from non-HCUs. This suggests that the HCU status tends to be driven by one or more major comorbidities, rather than CKD itself, which is also reflected in the sample baseline characteristics and our HCU prediction model. HCUs also have higher concentration of diagnoses within the top seven MDCs, which accounted for $75 \%$ of their admissions, compared with $65.8 \%$ in non-HCU, which implies a more focused catalogue of core health issues. While renal disease becomes a major factor driving hospitalisations when end-stage is reached, requiring renal replacement therapy, in pre-end stage CKD common

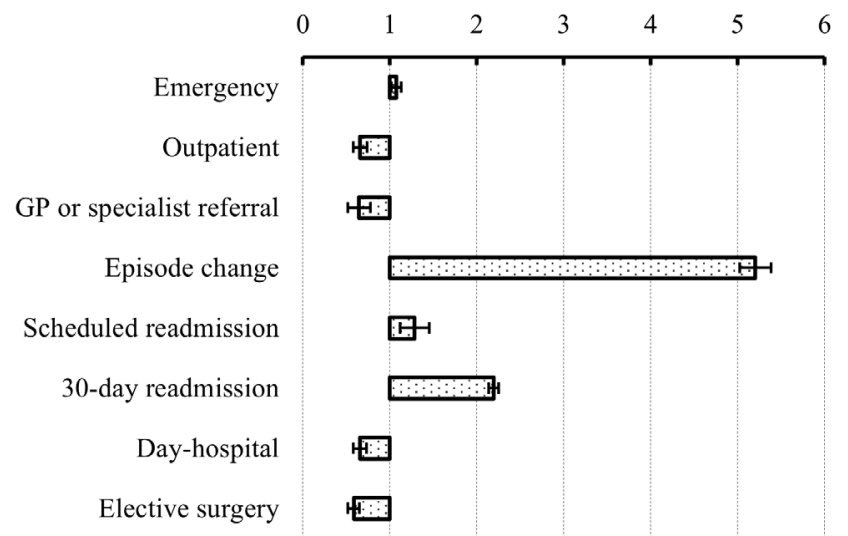

Figure 2 Relative risk of hospitalisation pathways in $\mathrm{HCU}$ versus non-HCU groups. HCU, high-cost user; GP, general practitioner. 
Table 3 Admission rates in top seven categories contributing to expenditures

\begin{tabular}{|c|c|c|c|}
\hline Category & Non-HCU & HCU & RR $(95 \% \mathrm{Cl})$ \\
\hline Any admission & 4479 & 2836 & \\
\hline \multicolumn{4}{|l|}{ MDC (\% of sample expenditure) } \\
\hline Kidney and urinary tract (15.3\%) & 826 & 489 & $0.93(0.83$ to 1.04$)$ \\
\hline $\begin{array}{l}\text { Musculoskeletal system and connective tissue } \\
(12.6 \%)\end{array}$ & 315 & 225 & 1.13 (0.96 to 1.29$)$ \\
\hline Factors influencing health status (8\%) & 207 & 251 & $1.92(1.74$ to 2.09$)$ \\
\hline Respiratory system (7.9\%) & 290 & 220 & $1.2(1.03$ to 1.37$)$ \\
\hline Nervous system (4.8\%) & 160 & 197 & 1.94 (1.74 to 2.15$)$ \\
\hline Other $(22.9 \%)$ & 1530 & 708 & $0.73(0.66$ to 0.81$)$ \\
\hline
\end{tabular}

Test statistic for equal distribution between groups: $\chi^{2}(21)=336.6996, p=0.000$.

Statistical significance denoted in bold for $p$-values $<0.05$.

$\mathrm{HCU}$, high-cost user; MDC, major diagnostic category; RR, relative risk.

reasons behind inpatient admissions are heart failure (31.7\% of circulatory system admissions), myoneural disorders (nervous system, 16.8\%), pneumonia (respiratory system, 28.2\%) and FIHS (rehabilitation, 45.8\%). Although the first three conditions are well known in patients with CKD, the forth common cause, FIHS, is less understood. According to ICD-10 codes Z00-Z99, FIHS consists of codes that are designated as the principal, or first listed, diagnosis in situations when a person with a resolving disease, injury or chronic condition requires specific aftercare. ${ }^{26}$ Some of the 16 Z-code categories, including counselling, follow-up, aftercare and miscellaneous codes, are directly applicable to management of admitted patients with CKD.

Emergency presentations leading to inpatient admissions are typically associated with high cost. ${ }^{10}$ While rates of emergency admissions were higher in HCU than nonHCU, the RR point estimate was 1.07 , suggesting only a small (albeit statistically significantly) increase. The implication of this was that there were other pathways leading HCUs to inpatient care. One possible interpretation is that heavy users' demand for inpatient care is somewhat predictable, demonstrated in our study by the rates of scheduled and 30-day readmissions, and also corroborated by lower rates of admissions by GP or specialist referral, from the outpatient department, or for elective procedures. In addition, episode change emerged as a major pathway accounting for $17 \%$ of episodes in HCUs, compared with $3 \%$ in the non-HCU group. A further inspection revealed that a high proportion of these admissions was for the purposes of rehabilitation of major and minor complexity, which was consistent with the increased RR of FIHS (45.8\% of admissions in this category, vs $4.4 \%$ in the non-HCU group).

The combination of FIHS as one of the major factors linked to admissions, and episode change as a common pathway in HCUs, suggests that, after the initial admission for traditional risk factors, costs are driven by conditions such as rehabilitation, counselling, follow-up and aftercare planning, associated with discordant comorbid conditions like cancer, dementia, arthritis, fractures and frailty. These conditions often demand more carer services, displacement from home, moving to transitional or residential aged care services and nursing home placements. Frailty is a state of increased vulnerability to physical stressors and is associated with increased risk of poor clinical outcomes, more so in patients with CKD compared with general population. This occurs due to a combination of degeneration of physiological systems, decline in psychological health and inadequate social support, which are all common risk factors in CKD. ${ }^{6}$ These discordant factors can be addressed in a multidisciplinary model of care, often involving other specialities like geriatrics and rehabilitation, which prevents prolonged hospitalisations. International societies have increasingly recognised these problems and developed guidelines under the broad term renal supportive care, although the primary focus of their application has so far been on end-stage disease. ${ }^{27}$

Our results suggest these models of care have an important role to play at earlier stages as well, and may enable strategies for reducing hospitalisations. Identifying the factors contributing to eventual high cost can direct these models of care in two ways. Firstly, by targeting the groups of patients who are likely to experience escalating healthcare needs, predictably leading to hospitalisations. Secondly, by allowing these programmes to focus on those areas of need where key improvements can be achieved, and hospitalisations averted. For example, an early intervention from allied health, involving occupational therapists and social workers, and offered to patients showing signs of FIHS, would seek to prevent 
prolonged hospitalisations by way of transfer of care to a residential or transitional care setting, and a nursing home placement. ${ }^{28} 29$

In considering which diagnoses and pathways can be linked to potentially preventable hospitalisations, one has to keep in mind the considerably higher death rate in the HCU group compared with the rest of the sample $(19.5 \%$ vs $2.7 \%)$. While proximity of death is a major factor contributing to healthcare costs, ${ }^{30}$ its application to explaining costs is retrospective by nature (which is why this variable was not part of our HCU prediction model), and many of the episodes taking place around the time of death may not be avoidable or amenable to secondary prevention.

Although exploratory in nature, our study has considerable strengths. The large pre-dialysis registry, linked with a comprehensive hospitalisations data set, provides an opportunity for a wide-ranging analysis of factors associated with patients with CKD who are admitted to hospitals. The data are collected in the first year of consent, when patients came into specialist care, rather than towards the end of progressive CKD journey known to be associated with higher costs. Still, our analyses inherited the limitations of the data sources, in terms of information available to researchers and data quality. Undiagnosed cases of CKD and patients not presenting to Queensland public renal practice clinics were not included in the CKD.QLD Registry and are, therefore, not represented in this study. This shortcoming was alleviated by the extensive network of participating renal practice clinics in Queensland. ${ }^{15}$ However, because the study enrolment places emphasis on advanced CKD that warrants a referral and selfselection to attend specialist care, its generalisability to a broader spectrum of CKD may be limited.

\section{CONCLUSION}

The episode change pathway and FIHS account for a large share of inpatient care in the HCU group, which makes them major candidate areas for averting admissions. Models of care that incorporate strategies to identify, assess and manage discordant comorbidities in a holistic, multidisciplinary method, and seek to address psychosocial risk factors, such as renal supportive care, have the potential to optimise costs and provide quality care for this vulnerable population.

Acknowledgements The authors would like to acknowledge site coordinators and staff contributing to the CKD.QLD Registry, and the Queensland Health Statistical Services Branch who extracted the linked data. The authors would like to thank the patients who made this study possible by consenting to the CKD.QLD Registry.

Contributors PMS, SKV, WEH, JZ, AC, HGH and LBC contributed to the study conception and design. PMS and LBC performed the statistical analyses. PMS, SKV and LBC drafted the manuscript. PMS, SKV, WEH, JZ, AC, HGH and LBC provided comments on drafts of the manuscript. PMS, SKV, WEH, JZ, AC, HGH and LBC read and approved the final manuscript.

Funding The study received funding support from the National Health and Medical Research Council (NHMRC) through the CKD Centre of Research Excellence (number 1079502).
Competing interests None declared.

Patient consent for publication Not required.

Ethics approval The study was carried out under the approval of the Royal Brisbane and Women's Hospital Human Research Ethics Committee, reference number HREC/15/QRBW/294.

Provenance and peer review Not commissioned; externally peer reviewed.

Data availability statement Data may be obtained from a third party and are not publicly available. The study analysed a linked dataset of CKD.QLD Registry and the Queensland Hospital Admitted Patient Data Collection (QHAPDC). The data are not publicly available but can be accessed by researchers upon request made to CKD. QLD and the Queensland Government Department of Health Statistical Services Branch, respectively.

Supplemental material This content has been supplied by the author(s). It has not been vetted by BMJ Publishing Group Limited (BMJ) and may not have been peer-reviewed. Any opinions or recommendations discussed are solely those of the author(s) and are not endorsed by BMJ. BMJ disclaims all liability and responsibility arising from any reliance placed on the content. Where the content includes any translated material, BMJ does not warrant the accuracy and reliability of the translations (including but not limited to local regulations, clinical guidelines, terminology, drug names and drug dosages), and is not responsible for any error and/or omissions arising from translation and adaptation or otherwise.

Open access This is an open access article distributed in accordance with the Creative Commons Attribution Non Commercial (CC BY-NC 4.0) license, which permits others to distribute, remix, adapt, build upon this work non-commercially, and license their derivative works on different terms, provided the original work is properly cited, appropriate credit is given, any changes made indicated, and the use is non-commercial. See: http://creativecommons.org/licenses/by-nc/4.0/.

\section{ORCID iDs}

P. Marcin Sowa http://orcid.org/0000-0001-8892-4133

Wendy E. Hoy http://orcid.org/0000-0002-8405-1539

\section{REFERENCES}

1 Kerr M, Bray B, Medcalf J, et al. Estimating the financial cost of chronic kidney disease to the NHS in England. Nephrol Dial Transplant 2012;27 Suppl 3:iii73-80.

2 Wyld MLR, Lee CMY, Zhuo X, et al. Cost to government and society of chronic kidney disease stage 1-5: a national cohort study. Intern Med J 2015;45:741-7.

3 Baumeister SE, Böger CA, Krämer BK, et al. Effect of chronic kidney disease and comorbid conditions on health care costs: a 10-year observational study in a general population. Am J Nephrol 2010;31:222-9.

4 Smith DH, Gullion CM, Nichols G, et al. Cost of medical care for chronic kidney disease and comorbidity among enrollees in a large HMO population. J Am Soc Nephrol 2004;15:1300-6.

5 Lee W-C, Lee Y-T, Li L-C, et al. The number of comorbidities predicts renal outcomes in patients with stage 3-5 chronic kidney disease. J Clin Med 2018;7:493.

6 Chen B, Fan VY, Chou Y-J, et al. Costs of care at the end of life among elderly patients with chronic kidney disease: patterns and predictors in a nationwide cohort study. BMC Nephrol 2017;18:36.

7 Tonelli M, Wiebe N, Guthrie B, et al. Comorbidity as a driver of adverse outcomes in people with chronic kidney disease. Kidney Int 2015;88:859-66.

8 Jha V, Garcia-Garcia G, Iseki K, et al. Chronic kidney disease: global dimension and perspectives. The Lancet 2013;382:260-72.

9 Purdey S, Huntley A. Predicting and preventing avoidable hospital admissions: a review. J R Coll Physicians Edinb 2013;43:340-4.

10 LaCalle E, Rabin E. Frequent users of emergency departments: the myths, the data, and the policy implications. Ann Emerg Med 2010;56:42-8.

11 van Walraven $C$, Bennett $C$, Jennings $A$, et al. Proportion of hospital readmissions deemed avoidable: a systematic review. CMAJ 2011;183:E391-402.

12 Morton RL, Kurella Tamura M, Coast J, et al. Supportive care: economic considerations in advanced kidney disease. Clin J Am Soc Nephrol 2016;11:1915-20.

13 Purtell L, Sowa PM, Berquier I, et al. The kidney supportive care programme: characteristics of patients referred to a new model of care. BMJ Support Palliat Care 2018. doi:10.1136/ bmjspcare-2018-001630. [Epub ahead of print: 04 Dec 2018]. 
14 Sowa PM, Purtell L, Hoy WE, et al. Utilization and costs of health care in a kidney supportive care program. J Palliat Care 2020;35:176-84.

15 Venuthurupalli SK, Hoy WE, Healy HG, et al. CKD.QLD: chronic kidney disease surveillance and research in Queensland, Australia. Nephrology Dialysis Transplantation 2012;27:iii139-45.

16 Queensland Health. Queensland data linkage framework. Brisbane: Queensland Government Department of Health, 2017.

17 Queensland Government Department of Health. Health funding principles and guidelines 2015-16 financial year. Queensland: Queensland Government Department of Health, 2016.

18 Wammes JJG, van der Wees PJ, Tanke MAC, et al. Systematic review of high-cost patients' characteristics and healthcare utilisation. BMJ Open 2018;8:e023113.

19 Australian Institute of Health and Welfare. Health expenditure Australia 2015-16. Canberra: Australian Government, 2017.

20 Jommi C, Armeni P, Battista M, et al. The cost of patients with chronic kidney failure before dialysis: results from the IRIDE observational study. Pharmacoecon Open 2018;2:459-67.

21 Eriksson JK, Neovius M, Jacobson SH, et al. Healthcare costs in chronic kidney disease and renal replacement therapy: a population-based cohort study in Sweden. BMJ Open 2016;6:e012062.

22 Manns B, Hemmelgarn B, Tonelli M, et al. The cost of care for people with chronic kidney disease. Can J Kidney Health Dis 2019;6:205435811983552.
23 Australian Institute of Health and Welfare. The health and welfare of Australia's Aboriginal and Torres Strait Islander peoples: 2015. AlHW; 2015.

24 Maisonneuve P, Agodoa L, Gellert R, et al. Distribution of primary renal diseases leading to end-stage renal failure in the United States, Europe, and Australia/New Zealand: results from an international comparative study. Am J Kidney Dis 2000;35:157-65.

25 Mallett A, Patel C, Salisbury A, et al. The prevalence and epidemiology of genetic renal disease amongst adults with chronic kidney disease in Australia. Orphanet J Rare Dis 2014;9:98.

26 International Statistical Classification of Diseases and Related Health Problems. 10th revision (ICD-10), who version for 2019, chapter XXI: factors influencing health status and contact with health services (Z00-Z99), 2019. Available: https://icd.who.int [Accessed 2 Jun 2020].

27 Davison SN, Levin A, Moss AH, et al. Executive summary of the KDIGO controversies conference on supportive care in chronic kidney disease: developing a roadmap to improving quality care. Kidney Int 2015;88:447-59.

28 Hopkins RB, Garg AX, Levin A, et al. Cost-Effectiveness analysis of a randomized trial comparing care models for chronic kidney disease. Clin J Am Soc Nephrol 2011;6:1248-57.

29 Menzin J, Lines LM, Weiner DE, et al. A review of the costs and cost effectiveness of interventions in chronic kidney disease: implications for policy. Pharmacoeconomics 2011;29:839-61.

30 Zweifel P, Felder S, Meiers M. Ageing of population and health care expenditure: a red herring? Health Econ 1999;8:485-96. 